Recepción: 18 / 01 / 2018

Aceptación: 09 / 04 / 2018

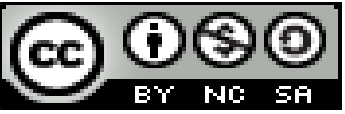

Ciencias de la Salud

Publicación: 01 / 08 / 2018

Artículo de investigación

\title{
Abordaje médico terapéutico en H1N1 y su impacto en el paciente
}

Therapeutic medical approach in H1N1 and its impact on the patient

\section{Abordagem médica terapêutica no H1N1 e seu impacto no paciente}

\author{
Mateo Fantoni-Añazco ${ }^{\text {I }}$ \\ mateojosuefantoni@gmail.com \\ Andrea Mera-Viteri II \\ ansomevi0607@outlook.es \\ Galo J. Vinueza-Aguay III \\ galojvinueza@uta.edu.ec
}

Correspondencia: mateojosuefantoni@gmail.com

${ }^{\text {I } M e ́ d i c o, ~ U n i v e r s i d a d ~ T e ́ c n i c a ~ d e ~ A m b a t o, ~ A m b a t o, ~ E c u a d o r . ~}$

II Médico, Universidad Técnica de Ambato, Ambato, Ecuador.

IIIDiploma Superior en Docencia en Ciencias de la Salud, Especialista en Medicina de Emergencias y Desastres, Doctor en Medicina y Cirugía, Universidad Técnica de Ambato, Ambato, Ecuador. 


\section{Resumen}

La influenza $\mathrm{H} 1 \mathrm{~N} 1$, ha sorprendido históricamente al mundo, ocasionando una sobrecarga en los servicios de atención médica, así como, problemas de orden económico, social y de seguridad. La situación actual es marcadamente diferente, ya que si bien se ha advertido a la salud pública; esto ha permitido proporcionar una oportunidad sin precedente para prepararse para una pandemia y desarrollar las capacidades necesarias en cada país para enfrentarla y atenuar los efectos de orden social. En el Ecuador, el Ministerio de Salud Pública con el apoyo de la Organización Mundial de la Salud ha desarrollado un plan de contingencia para una posible pandemia de influenza, así como protocolos en las áreas de vigilancia epidemiológica, servicios de salud, salud ambiental, laboratorio, salud mental, comunicación social, entre otros. A continuación, se presenta un relato de caso de un paciente joven que debuta con las manifestaciones clínicas clásicas de la influenza H1N1 que desenvolvió complicaciones respiratorias graves y por medio de un soporte terapéutico adecuado y manejo multidisciplinar ha permitido una evolución favorable del paciente; por lo que se empleó un estudio de caso que permitió concluir que la prontitud del paciente al acercase a un centro de salud una vez diagnosticado es asertivo, sin embargo, en el caso acordado el retardo accionar del paciente permitió la incubación del virus conllevando a un tratamiento médico.

Palabras clave: influenza; tratamiento; paciente.

\section{Abstract}

H1N1 influenza has historically surprised the world, causing an overload in health care services, as well as economic, social and security problems. The current situation is markedly different, since although public health has been warned; this has provided an unprecedented opportunity to prepare for a pandemic and develop the necessary capacities in each country to confront and mitigate the effects of social order. In Ecuador, the Ministry of Public Health with the support of the World Health Organization has developed a contingency plan for a possible influenza pandemic, as well as protocols in the areas of epidemiological surveillance, health services, environmental health, laboratory, mental health, social communication, among others. The following is a case report of a young patient who debuted with the classic clinical manifestations of influenza H1N1 who developed severe respiratory complications and through an adequate therapeutic support and multidisciplinary management has allowed a favorable evolution of the 
patient; Therefore, a case study was used to conclude that the patient's readiness to approach a health center once diagnosed is assertive, however, in the agreed case, the patient's delay allowed the incubation of the virus leading to a medical treatment.

Keywords: influenza; treatment; patient.

\section{Resumo}

A gripe $\mathrm{H} 1 \mathrm{~N} 1$ historicamente tem surpreendido o mundo, causando uma sobrecarga nos serviços de saúde, bem como problemas econômicos, sociais e de segurança. A situação atual é marcadamente diferente, uma vez que, embora a saúde pública tenha sido advertida; Isso proporcionou uma oportunidade sem precedentes de se preparar para uma pandemia e desenvolver as capacidades necessárias em cada país para enfrentar e mitigar os efeitos da ordem social. No Equador, o Ministério da Saúde Pública, com o apoio da Organização Mundial da Saúde desenvolveu um plano de contingência para uma possível pandemia de gripe e protocolos nas áreas de vigilância epidemiológica, serviços de saúde, saúde ambiental, laboratório, saúde mental, comunicação social, entre outros. Em seguida, um relato de caso de um paciente jovem que estreia com as manifestações clínicas clássicas da gripe H1N1 complicações respiratórias graves e desdobrou usando um suporte terapêutico adequado e apresenta tratamento multidisciplinar permitiu uma evolução favorável do paciente; assim que um estudo de caso permitiu concluir que a disposição do paciente para chegar perto de um centro de saúde uma vez diagnosticada é foi usado assertivo, no entanto, no caso concordaram as ações atrasar o paciente permitido à incubação do vírus levando a um tratamento médico.

Palavras chave: influenza; tratamento; paciente.

\section{Introducción}

El virus de la Influenza A H1N1 pertenece a la familia de los Orthomyxoviridae, el cual está compuesto por un genoma de 8 segmentos de ARN de sentido negativo que va a codificar 11 proteínas (Sullivan, Jacobson, Dowdle \& Poland, 2010). El virus sufrió un reordenamiento en el año 2009, llegando a estacionarse como pandemia y actualmente se posiciona como una enfermedad estacional en los meses de enero a abril en Ecuador (Ministerio de Salud Pública de Ecuador, 2016). Su capacidad infecciosa radica en el conglomerado de la gripe aviar, porcina y 
humana (Adeola, Olugasa, \& Emikpe, 2017). Respecto a Ecuador, en el 2015 se hallaron 116 casos con una muerte y el 1\% de letalidad. En el año 2016 existió un rebrote de 950 casos con 83 muertes y 9\% de letalidad (Organización Panamericana de la Salud, 2018). En los 10 primeros meses del año 2017 se notificaron 306 casos de influenza de los cuales 5 fueron confirmados de H1N1 (Ministerio de Salud Pública de Ecuador, 2016). El virus se trasmite por medio del aire de los cerdos a los humanos, lo que implica que el consumo de su carne no lo transmite; el contagio de humano a humano es por medio del contacto cercano por la erupción de saliva al hablar. Los pacientes inmunodeprimidos son los más susceptibles a infecciones, por lo que desde su aparición la OMS distribuyó las vacunas a la mayor parte de países; sin embargo, fueron los gobiernos los encomendados en distribuir a los que consideran como grupos prioritarios; en el caso de nuestro país fue a mujeres embarazadas, inmunodeprimidos, niños menores de 6 meses, adultos mayores y al personal de salud (Cárdenas et al., 2014).

Las manifestaciones clínicas comunes pueden ir desde tos, fiebre mayor de $38^{\circ} \mathrm{C}$, rinorrea, dolor de garganta y dolores musculares; y, entre los síntomas menos comunes se hallan las molestias articulares y abdominales (Ministerio de Salud Pública de Ecuador, 2016). El diagnóstico se realiza a través del hisopado de la orofaringe, pero muchas de las veces han dado falsos negativos sobre todo cuando el virus se aledaña en las vías respiratorias inferiores y allí es necesario la broncoscopia o el examen de oro denominado prueba RT-PCR o reacción en cadena de polimerasa-transcriptasa inversa en tiempo real. En cuanto al tratamiento se utilizan los inhibidores de la neuraminidasa como son el oseltamivir y zanamivir que impiden la propagación del virus a las demás células sanas (Zimmer y Burke, 2009).

\section{Descripción del caso}

Paciente masculino de 34 años de edad, mestizo, instrucción secundaria, nacido en Ambato y residente en la parroquia Picaihua (caserío San Pedro) cantón Ambato, provincia de Tungurahua. Estado civil casado, ocupación panificador, lateralidad diestra, grupo sanguíneo ORH+. El mencionado paciente no refiere antecedentes patológicos personales, antecedente quirúrgico de apendicectomía hace 6 años y antecedente patológico familiar, el padre fallece con diabetes Mellitus. 
El paciente que acude por presentar hace aproximadamente tres días rinorrea hialina acompañado de alza térmica no cuantificada sin causa aparente, por lo que acude a facultativo quien prescribe Paracetamol + Norfloxacina, sin presentar mejoría, posterior a lo cual presenta disnea de medianos esfuerzos cambiándose de medicación a Sultamicilina; sin embargo, el cuadro empeora hace 48 horas cuando presenta esputo hemoptoico en poca cantidad acompañado de cianosis y disnea de pequeños esfuerzos, conllevado a acudir a emergencia de esta casa de salud, presentando los siguientes signos vitales de TA: 112/68 mmHg, FC: 861pm, FR: 25rpm, Sat O2: 73\%, ECG: 15/15 (O4V5M6).

Al examen físico de emergencia se halla consciente, afebril, ojos con pupilas isocóricas, reactivas a la luz, sin signos de focalidad ni lateralidad neurológica. Boca con mucosas orales húmedas, pulmones con murmullo vesicular disminuido y la presencia de estertores bilateral, taquipneico con retracciones subcostales, corazón rítmico sin ruidos sobreañadidos; abdomen suave, depresible, no doloroso a la palpación, presencia de ruidos hidroaéreos. Extremidades simétricas sin edema; bajo estos antecedentes el diagnóstico presuntivo se enfoca a Neumonía Grave + Sospecha de AH1N1, ante lo cual se solicita los siguientes exámenes complementarios: Pruebas de biometría hemática, bioquímica y serología.

\section{Tabla 1}

Resultado de biometría hemática, bioquímica sanguínea y serología del paciente en estudio

\begin{tabular}{lll}
\hline & \multicolumn{1}{c}{ Resultado } & $\begin{array}{l}\text { Valor } \\
\text { referencia }\end{array}$ \\
\multicolumn{2}{l}{ Biometría Hemática } & \\
Leucocitos & $4560 \mathrm{k} / \mathrm{ul}$ & $4800-10800 \mathrm{k} / \mathrm{ul}$ \\
Monocitos & $0,1 \%$ & $1.9-9.0 \%$ \\
Eosinófilos & $0,6 \%$ & $1.0-5.0 \%$ \\
Basófilos & $0,1 \%$ & $0.2-1.0 \%$ \\
Hematíes & $5,31 \mathrm{M} / \mathrm{ul}$ & $4.50-6.00 \mathrm{M} / \mathrm{ul}$ \\
Hemoglobina & $15,20 \mathrm{gr} / \mathrm{dl}$ & $14.00-18.00 \mathrm{gr} / \mathrm{dl}$ \\
Hematocrito & $47,4 \%$ & $41.0-51.0 \%$ \\
Plaquetas & $119 \mathrm{~K} / \mathrm{ul}$ & $150-450 \mathrm{~K} / \mathrm{ul}$ \\
Bioquímica & & \\
Urea & $30 \mathrm{mg} / \mathrm{dl}$ & $10.0-50.0 \mathrm{mg} / \mathrm{dl}$ \\
Creatinina & $1,07 \mathrm{mg} / \mathrm{dl}$ & $0.7-1.3 \mathrm{mg} / \mathrm{dl}$ \\
\hline
\end{tabular}




$\begin{array}{lcc}\begin{array}{l}\text { Glucosa } \\ \text { Serología }\end{array} & 123,12 \mathrm{mg} / \mathrm{dl} & 70-100 \mathrm{mg} / \mathrm{dl} \\ \text { PCR } & 87.3 \mathrm{mg} / \mathrm{dl} & 0.0-5.0 \mathrm{mg} / \mathrm{dl}\end{array}$

Fuente: Laboratorio clínico Hospital Provincial Docente Ambato (año).

\section{Biometría hemática y bioquímica}

Presencia de una leve leucopenia, sin otras alteraciones considerables.

\section{Serología}

Evidencia de proteína $\mathrm{C}$ reactiva indicativo de reactante de fase aguda, siendo este un proceso inflamatorio a nivel del parénquima pulmonar y razón por la que se eleva su valor.

\section{Prueba Radiográfica}

En la radiografía anteroposterior de tórax se evidencia la presencia un patrón alveolar con aspecto algodonoso con tendencia a la coalescencia y broncograma aéreo, que orienta al diagnóstico de neumonía (Figura N.1).

\section{Figura 1}

Radiografía anteroposterior de tórax donde se evidencia patrón alveolar de aspecto algodonoso con tendencia a la coalescencia.

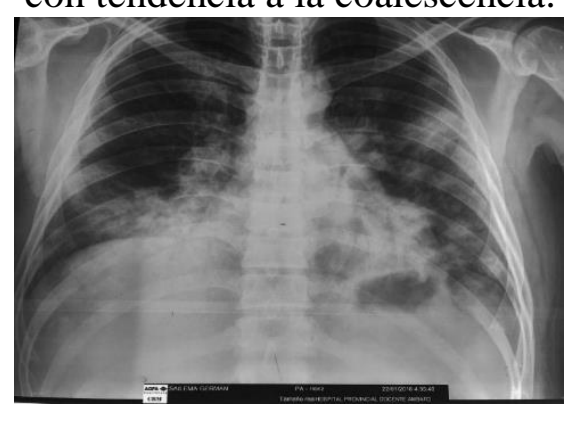

Fuente: Departamento de Radiología del Hospital Provincial Docente Ambato (año).

En el área de Emergencia se administra el siguiente tratamiento:

- Hidratación al paciente (Solución Salina 0.9\% 1000cc a 60cc/h).

- Oxigenoterapia por mascarilla de alto flujo a 15 litros. 
- Nebulizaciones (2cc Dexametasona + 2cc de Solución Salina cada 20 minutos por 4 ocasiones; y, después cada 6 horas)

- Oseltamivir $150 \mathrm{mg}$ VO c/12h por 5 días.

- Antibioticoterapia (ceftriaxona 2gr IV c/12h + claritromicina 500mg VO c/12h

- Hidrocortisona 500mg IV c/8h

- Aislamiento respiratorio

- Se le solicita el hisopado nasofaríngeo para AH1N1.

El paciente ingresa a aislamiento al día siguiente, con oxígeno por mascarilla a 12 litros por minuto con saturación del 80\%, FR: 26-28rpm, TA: 110/70 mmHg, FC: 781pm. Al examen físico despierto, lucido, consciente, orientado, taquipneico, afebril; mucosas orales húmedas, tórax con retracciones intercostales moderadas, pulmones con murmullo vesicular disminuido y presencia de estertores diseminados en ambos campos pulmonares, corazón rítmico sin ruidos sobreañadidos; abdomen suave, depresible, no doloroso a la palpación, ruidos hidroaéreos presentes. Se continua con hidratación + oxigenoterapia por mascarilla de alto flujo + antibioticoterapia + protector gástrico $($ omeprazol $)+$ nebulizaciones + corticoesteroides. Se recibe el resultado del hisopado nasofaríngeo para H1NI siendo este POSITIVO.

Posteriormente, el paciente ingresa a la Unidad de Cuidados Intensivos con diagnóstico de Neumonía Grave de tipo Influenza, tras dos días de permanecer en esta área se realiza sedo analgesia presentando los siguientes signos vitales TA 140/110mmHg, TAM 98-79 mmHg, FC: 90-701pm, FR: 24-47rpm, SatO2,: 82-91\% con mascarilla de alto flujo, temperatura: $36^{\circ} \mathrm{C}-37^{\circ} \mathrm{C}$, Glasgow con efectos de sedo analgesia 3t/15 (O1V1M1), pupilas isocóricas de $2 \mathrm{~mm}$, reflejo corneal presente, sonda orogástrica por la cual recibe nutrición enteral con buena tolerancia, presenta enfisema subcutáneo en región del cuello y tórax, oroentubado acoplado a ventilación mecánica, cuello sin ingurgitación, tórax simétrico, expansible, corazón normofonético, pulmones con murmullo vesicular disminuido, estertores bilaterales. Abdomen suave, depresible, no doloroso a la palpación, sonda vesical permeable, diuresis clara con buenos volúmenes de diuresis, extremidades simétricas sin edema. 
Se le realizan nuevos exámenes complementarios, a saber: Biometría hemática, perfil lipídico y serología, presentando Paciente que presenta marcada linfopenia, hemoglobina disminuida con indicativo para anemia leve, PCR que ha aunado considerablemente, sin otros resultados de relevancia.

\section{Tabla 2}

Resultado de biometría hemática, bioquímica sanguínea, perfil lipídico y serología del paciente en estudio.

\section{Resultado Valor de referencia}

\section{Biometría Hemática}

$\begin{array}{lll}\text { Leucocitos } & 5640 \mathrm{k} / \mathrm{ul} & 4800-10800 \mathrm{k} / \mathrm{ul} \\ \text { Neutrófilos } & 93.3 \% & 43.0-65.0 \% \\ \text { Eosinófilos } & 0.4 \% & 1.0-5.0 \% \\ \text { Basófilos } & 0.1 \% & 0.2-1.0 \% \\ \text { Linfocitos } & 4.0 \% & 20.5-45.5 \% \\ \text { Monocitos } & 1.9 \% & 1.9-9.0 \% \\ \text { Hematíes } & 4,47 \mathrm{M} / \mathrm{ul} & 4.50-6.00 \mathrm{M} / \mathrm{ul} \\ \text { Hemoglobina } & 12.40 \mathrm{gr} / \mathrm{dl} & 14.00-18.00 \mathrm{gr} / \mathrm{dl} \\ \text { Hematocrito } & 41.3 \% & 41.0-51.0 \% \\ \text { Plaquetas } & 337 \mathrm{~K} / \mathrm{ul} & 150-450 \mathrm{~K} / \mathrm{ul} \\ \text { Bioquímica } & & \\ \text { Urea } & 57.4 \mathrm{mg} / \mathrm{dl} & 10.0-50.0 \mathrm{mg} / \mathrm{dl} \\ \text { Creatinina } & 0.6 \mathrm{mg} / \mathrm{dl} & 0.7-1.3 \mathrm{mg} / \mathrm{dl} \\ \text { Glucosa } & 155,6 \mathrm{mg} / \mathrm{dl} & 70-100 \mathrm{mg} / \mathrm{dl} \\ \text { Bilirrubina directa } & 0.28 \mathrm{mg} / \mathrm{dl} & 0.0-0.3 \mathrm{mg} / \mathrm{dl} \\ \text { Bilirrubina total } & 0,4 \mathrm{mg} / \mathrm{dl} & 0.0-1.10 \mathrm{mg} / \mathrm{dl} \\ \text { Albúmina } & 2.35 \mathrm{gr} / \mathrm{dl} & 3.50-4.90 \mathrm{gr} / \mathrm{dl} \\ \text { Globulinas } & 2,58 \mathrm{gr} / \mathrm{dl} & 2.30-3.50 \mathrm{gr} / \mathrm{dl} \\ \text { AST-TGO } & 51 \mathrm{U} / \mathrm{l} & 0-38 \mathrm{U} / \mathrm{l} \\ \text { ALT-TGP } & 62 \mathrm{U} / \mathrm{l} & 5-42 \mathrm{U} / 1 \\ \text { LDH } & 487 \mathrm{U} / \mathrm{l} & 240-480 \mathrm{U} / \mathrm{l} \\ \text { Fosfatasa alcalina } & 196 \mathrm{U} / \mathrm{l} & 40-129 \mathrm{U} / \mathrm{l} \\ \text { Amilasa } & 108 \mathrm{U} / \mathrm{l} & 28.0-100.0 \mathrm{U} / \mathrm{l}\end{array}$




\begin{tabular}{lll}
\hline Perfil Lipídico & & \\
Lipasa & $57.6 \mathrm{U} / \mathrm{l}$ & $13.0-60.0 \mathrm{U} / \mathrm{l}$ \\
Triglicéridos & $130 \mathrm{mg} / \mathrm{dl}$ & $50-200 \mathrm{mg} / \mathrm{dl}$ \\
Colesterol & $92 \mathrm{mg} / \mathrm{dl}$ & $140-200 \mathrm{mg} / \mathrm{dl}$ \\
HDL Colesterol & $27 \mathrm{mg} / \mathrm{dl}$ & $35-55 \mathrm{mg} / \mathrm{dl}$ \\
LDL Colesterol & $52 \mathrm{mg} / \mathrm{dl}$ & $0-150 \mathrm{mg} / \mathrm{dl}$ \\
Calcio & $7.30 \mathrm{mg} / \mathrm{dl}$ & $8.40-10.20 \mathrm{mg} / \mathrm{dl}$ \\
Serología & & \\
PCR & $20 \mathrm{mg} / \mathrm{dl}$ & $0.0-5.0 \mathrm{mg} / \mathrm{dl}$ \\
\hline
\end{tabular}

Fuente: Laboratorio clínico Hospital Provincial Docente Ambato (año).

\section{Prueba de uro análisis}

\section{Tabla 3}

Uroanálisis del paciente en estudio

\begin{tabular}{|c|c|c|}
\hline & Resultado & $\begin{array}{l}\text { Valor } \\
\text { referencia }\end{array}$ \\
\hline \multicolumn{3}{|l|}{ Uroanálisis } \\
\hline Color & Amarillo & \\
\hline Aspecto & Lig. Turbio & \\
\hline Densidad & 1.015 & $1.005-1.025$ \\
\hline $\mathrm{pH}$ & 6.00 & $5.00-7.00$ \\
\hline Leucocitos & NEGATIVO & \\
\hline Nitritos & NEGATIVO & \\
\hline Proteínas & NEGATIVO & \\
\hline Sangre & ++ & \\
\hline Glucosa & NEGATIVO & $0-0$ \\
\hline Cetonas & NEGATIVO & \\
\hline Urobilinógeno & NEGATIVO & \\
\hline Bilirrubina & NEGATIVO & \\
\hline Piecitos en orina & $0-1$ & $0.00-5.00$ \\
\hline $\begin{array}{l}\text { Hematíes en } \\
\text { orina }\end{array}$ & $18-20$ & $0.00-4.00$ \\
\hline Cel. Ept. Bajas & $0-1$ & $0.00-5.00$ \\
\hline Bacterias & Escasas & \\
\hline
\end{tabular}

Fuente: Laboratorio clínico Hospital Provincial Docente Ambato 


\section{Prueba gasométrica}

\section{Tabla 4}

Gasometría arterial del paciente en estudio

\begin{tabular}{ll}
\hline \multicolumn{2}{c}{ Gesultado } \\
pH & \multicolumn{1}{c}{$\begin{array}{c}\text { Rasotría } \\
\text { Arterial }\end{array}$} \\
$\mathrm{PO} 2$ & 7.423 \\
$\mathrm{PCO} 2$ & $49.1 \mathrm{mmHg}$ \\
$\mathrm{SO} 2$ & $40.8 \mathrm{mmHg}$ \\
$\mathrm{Hct}$ & $81.4 \%$ \\
$\mathrm{PCO} 2 \mathrm{t}$ & $31.5 \%$ \\
$\mathrm{cHCO} 3$ & $40.8 \mathrm{mmHg}$ \\
$\mathrm{BE}$ & $26.0 \mathrm{mmol} / \mathrm{L}$ \\
$\mathrm{SO} 2(\mathrm{c})$ & $1.5 \mathrm{mmol} / \mathrm{L}$ \\
& $85.5 \%$
\end{tabular}

Fuente: Laboratorio clínico Hospital Provincial Docente Ambato

Gasometría: Se aprecia un trastorno mixto que es una Acidosis respiratoria + Alcalosis metabólica debido a que el valor del pH se encuentra en el rango normal y la PCO2 y HCO3 están elevados.

Examen Radiográfico: En la radiografía anteroposterior de tórax se evidencia enfisema subcutáneo, además infiltrado algodonoso distribuido en ambos campos pulmonares, presencia de neumotórax izquierdo (Figura N.2).

\section{Figura 2}

Infiltrado algodonoso distribuido en ambos campos pulmonares, neumotórax izquierdo

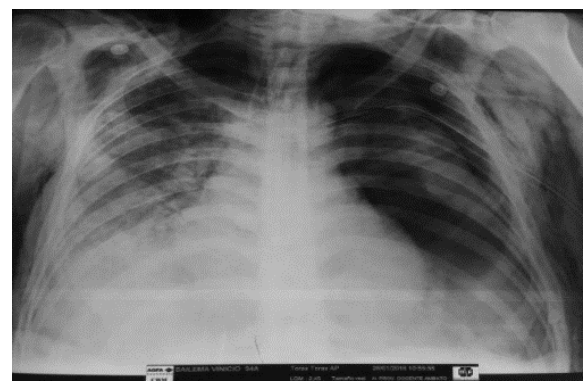

Fuente: Departamento de Radiología del Hospital Provincial Docente Ambato 
Paciente que ante la evidencia de neumotórax izquierdo se le coloca tubo torácico en hemitórax, obteniéndose la salida de aire y liquido serohemático de aproximadamente $30 \mathrm{cc}$. Recibe cobertura con betalactámicos (Imipenem + Cilastatina 1gr IV c/6h) + quinolonas (Levofloxacina 500mg IV c/12h) + macrólidos (azitromicina 1gr por sonda nasogástrica QD) + antivirales (oseltamivir 150mg por sonda nasogástrica c/12h) + corticoide (metilprednisolona 125mg IV c/8h) + antiemético(metoclopramida 10mg IV c/8h)+ mucolítico ( $\mathrm{N}$-acetilcisteína)+ antipirético (metamizol 2g IV) + Rocuronio 25mg IV + Sulfato de Magnesio 10cc IV QD+ Ácido ascórbico 1gr IV c/8h + Colchicina 1 tableta por sonda nasogástrica $\mathrm{c} / 12 \mathrm{~h}+$ Heparina de bajo peso molecular 50mg subcutáneo cada 12 horas +Lágrimas artificiales 1 gota cada 6 horas.

Paciente que permanece hemodinámicamente estable sin apoyo de inotrópico positivo, continúa con soporte ventilatorio y antibioticoterapia.

Ante la sospecha de una neumonía por influenza AH1N1 hay que tomar en cuenta que puede ser netamente de etiología viral o también el virus se asocia concomitantemente con bacterias y el principal agente de asociación es el Streptococo pneumoniae y en menor cuantía el Staphylococcus aureus, el Streptococcus pyogenes, Streptococcus mitis y Haemophilus influenzae; sin embargo, independientemente de su asociación o no, produce las mismas manifestaciones clínicas y llegan a producir una insuficiencia respiratoria grave (You, Huang, Chen, Chang, Liao \& Huang, 2017). En el caso de nuestro paciente hay una combinación de neumonía bacteriana y viral; lo que indica que el tratamiento debe ser a base de antivirales y antibióticos; es por ello que estudios indican que el tratamiento de la influenza AH1N1 está aprobado tanto porosetalmivir y el zanamivir que son inhibidores de la neuraminidasa; es decir, inhiben las glucoproteínas que se hallan en la envoltura del virión impidiendo así que se siga diseminando grave (You et al., 2017). Según estudios la dosis de oseltamivir es de 75mg VO BID por 5 días a las 48 horas de haberse presentado los síntomas; sin embargo, cuando se trata de una neumonía grave la dosis debe incrementarse a $150 \mathrm{mg}$ c/12h, pero si existe un clearance de creatinina menor o igual de 30ml/min deberá reducirse a $75 \mathrm{mg}$ al día. (Sullivan et al., 2010) Por tanto, el manejo de nuestro paciente está siendo adecuado conforme a la literatura ya que posee una preservada función renal y al tratarse de una neumonía grave requería de $150 \mathrm{mg}$ c/12h. En contraparte, el paciente no mejoró prontamente debido a que cuando acudió a la casa de salud 
habían pasado ya 72 horas del proceso viral y el tratamiento con oseltamivir es efectivo dentro de las primeras 48 horas de haberse presentado los síntomas.

En ciertos pacientes ha existido resistencia a los medicamentos antivirales y de allí surge el incremento del número de casos a nivel mundial y nacional; esta resistencia no se debe a que estos fármacos hayan dejado de ser eficaces por el contrario la resistencia tiene su base en la administración inadecuada de dosis tanto para la profilaxis como del tratamiento; lo que lleva a que la exposición prolongada a osetalmivir produzca la mutación His275Tyr en la neuraminidasa viral lo que desemboca en mayor fortaleza del agente (Bautista, Chotpitayasunondh, Gao, Shaw \& Uyeki, 2010).

En el paciente en estudio es poco probable que haya existido una resistencia como la ya mencionada puesto que él fue tratado con la dosis y tiempo indicado; sin extensión a mayor número de días por lo que no tuvo una exposición prolongada a inhibidores de la neuraminidasa ni antes ni durante la patogenia. Ante esta problemática mundial ya existen estudios de ácido gálico que interrumpen la actividad virológica de la influenza A y B; la combinación con terapias adyuvantes dirigidas como inmunomoduladores e inmunoterapia se hallan en estudio para un mejor manejo (You et al, 2017).

Por otro lado, un estudio comparativo entre los pacientes con H1N1 en el año 2009, en relación con pacientes que padecieron H1N1 en el año 2017 halló que la comorbilidad de insuficiencia renal crónica era del 3\% y del 9\% de los casos, respectivamente. El ascenso del porcentaje es debido a que se ha comprobado la mutación del gen D222N en la hemaglutina A (componente del virus), caracterizada por el reemplazo del aminoácido ácido aspártico (D) por asparagina (N) en la posición 222; esto hace que el virus tenga mayor tropismo por el glomérulo renal causando su destrucción (Riquelme, Rioseco, Velásquez, Campos, Gayan \& Medina, 2017) y (Aceituno, Fica, Fasce, Andrade, \& Díaz, 2017).

Además, se ha demostrado que el virus tiene la capacidad de atravesar la barrera hematoencefálica inmadura y es por ello que la epilepsia, encefalopatía y encefalitis se ve en mayor proporción en niños que en adultos con H1N1 (Cárdenas et al, 2014) y (Riquelme, et al, 2017). Asimismo, es importante estar atentos a complicaciones aun no observadas en el paciente pero que deben ser consideradas en el manejo del mismo con la administración de sulfato de 
magnesio que disminuye la excitabilidad neuronal; por ende, menor la probabilidad epileptogénica (Aceituno, Fica, Fasce, Andrade, \& Díaz, 2017).

A futuro, estudios establecen que el virus de la H1N1 genera tres tipos primordiales de daño a nivel pulmonar que son lesión alveolar difusa, bronquiolitis necrotizante y hemorragia pulmonar generalizada lo que conlleva a que posterior a padecer la enfermedad si el paciente sobrevive tenga un síndrome respiratorio agudo severo, un trastorno ventilatorio obstructivo o un trastorno ventilatorio restrictivo (Liu, Peng, Liu \& Hua, 2015). Estos aspectos deben estar claros en la cinemática del manejo del paciente para tener presente las posibles complicaciones posteriores a su recuperación.

\section{Conclusiones}

El pronóstico de estos pacientes es bueno cuando han acudido al personal de salud dentro de las primeras 48 horas de haber presentado los síntomas. En el presente paciente ese resulta el primer problema, puesto que se inicia tardíamente el tratamiento, cuando ya ha pasado la etapa de incubación del virus, y requiere un tratamiento ante la infección del mismo; por lo que se da los dos tratamientos.

La detección en nuestro país la mayor parte de veces se limita al hisopado de orofaringe por la demanda en hospitales públicos, lo que puede generan falsos negativos; aumentando así el riesgo de mortalidad al no ser diagnosticados por broncoscopia, cultivos o RT-PCR; además el uso de oseltamivir lo provee el estado y en muchas ocasiones no alcanza para los pacientes en situación de riesgo y para los pacientes ya infectados.

Es importante tener presente que la supervivencia ante este proceso no significa una recuperación total; por el contrario, el paciente puede presentar limitaciones secuenciales como la fibrosis pulmonar o procesos obstructivos o restrictivos pulmonares; además, la mutación del gen no garantiza que el hecho de haberse vacunado no lo haga propenso a una nueva infección en el futuro. 


\section{Referencias Bibliográficas}

Sullivan, S., Jacobson, R., Dowdle, W \& Poland, G. (2010) H1N1 Influenza. Mayo Revista Clinic Procedings. 2010. 85(1):64-76.

Ministerio de Salud Pública de Ecuador. (2016). Influenza Ecuador Se. 2015. [en línea], Disponible en: www.salud.gob.ec/wp-content/uploads/2016/03/Boletín-Influenza-SE-43.pdf.

Adeola, O., Olugasa, \& Emikpe, B. (2017). Molecular detection of influenza A (H1N1) pdm09 viruses with $\mathrm{M}$ genes from human pandemic strains among Nigerian pigs, 2013-2015: implications and associated risk factors. Review Epidemiology and Infection. 145(16):33453360.

Organización Panamericana de la Salud (2018).Actualización epidemiológica SE 492017 - SE 02 2018. 2017. [en línea], Disponible en: https://www.paho.org/hq/index.php?option=com_docman\&task=doc_view\&Itemid=270\&gid=44 499\&lang=es

Cárdenas, G., Soto-Hernández, J., Díaz-Alba, A., Ugalde, Y., Mérida-Puga, J \& Rosetti, M (2014). Neurological events related to influenza A (H1N1) pdm09. Review Influenza and Other Respiratory Viruses. 8(3):339-346.

Zimmer, S y Burke, D. (2009). Historical Perspective - Emergence of Influenza A (H1N1) Viruses. The New England Journal Of Medicine. 2009 July; 361(3).

You, H., Huang, C., Chen, C., Chang, C., Liao, P \& Huang, S. (2017). Anti-pandemic influenza A (H1N1) virus potential of catechin and gallic acid. Journal of the Chinese Medical Association. Volume 81, Issue 5. Pp. 458-468. [En línea], Disponible en: https://doi.org/10.1016/j.jcma.2017.11.007.

Bautista, E., Chotpitayasunondh, T., Gao, H.S., Shaw, M \& Uyeki, T. (2010) Clinical Aspects of Pandemic 2009 Influenza A (H1N1) Virus Infection. Review Medical Progress [en línea], Disponible en: DOI: 10.1056/NEJMra1000449 
Riquelme, R., Rioseco, M., Velásquez, K., Campos, F., Gayan, B \& Medina, C. (2017). Cambios en la presentación clínica de la influenza A H1N1 pdm09 después de la pandemia. Rev Med Chile. Agosto.145 (980-986).

Aceituno, D., Fica, A., Fasce, R., Andrade, W \& Díaz, C. (2017). Glomerulonefritis aguda con requerimiento de diálisis asociada a influenza A H1N1pdm09: Comunicación de dos casos. Revista chilena de infectología. 34(1):81-86.

Liu, W., Peng, L., Liu, H \& Hua, S. (2015). Pulmonary Function and Clinical Manifestations of Patients Infected with Mild Influenza A Virus Subtype H1N1: A One-Year Follow-Up. PLOS ONE. 2015; 10(7):e0133698. 